\title{
Estado actual de la formación de Doctores en Ciencias de la Salud
}

\author{
Present situation of the formation of Doctors of Philosophy \\ in health sciences
}

\section{Ileana Elena Castañeda Abascal, ${ }^{\text {I }}$ Pastor Castell-Florit Serrate, ${ }^{\text {II }}$ Aida Rodríguez Cabrera, ${ }^{\text {III }}$ Leticia Fernández Garrote, ${ }^{\text {IV }}$ Magaly Iglesia Duquesne," Alcides Ochoa Alonsovi}

\footnotetext{
I Especialista de II Grado en Bioestadística. Doctor en Ciencias de la Salud. Profesor Titular. Escuela Nacional de Salud Pública. La Habana, Cuba.

II Doctor en medicina. Doctor en Ciencias. Escuela Nacional de Salud Pública. La Habana, Cuba.

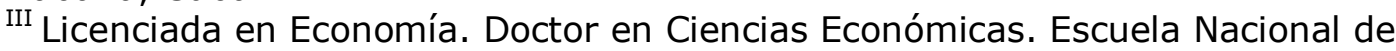
Salud Pública. La Habana, Cuba.

IV Especialista de II Grado en Bioestadística. Doctor en Ciencias de la Salud. Profesor Titular. Escuela Nacional de Salud Pública. La Habana, Cuba.

${ }^{\vee}$ Doctor en Medicina. Doctor en Ciencias Médicas. Escuela Nacional de Salud Pública. La Habana, Cuba.

${ }^{V I}$ Doctor en Medicina. Doctor en Ciencias de la Salud. Profesor Titular. Escuela Nacional de Salud Pública. La Habana, Cuba.
}

\section{RESUMEN}

Objetivo Identificar los factores favorables y dificultades que existen en la formación de doctores en ciencias de la salud.

Métodos Se realizó un estudio exploratorio mediante entrevistas en profundidad a 22 representantes de los Consejos Científicos de la Red de Centros Colaboradores de la Escuela Nacional de Salud Pública, para la formación de doctores en ciencias de la salud de instituciones de La Habana y de las Universidades Médicas de Santiago de Cuba y Camagüey. Se efectúo una lluvia de ideas con representantes de los centros de la red. 
Resultados Como elementos favorables están la existencia de una masa crítica de doctores en ciencias relacionados con la salud pública, disponibilidad de profesionales con potencialidad para alcanzar el grado científico, consolidación del Sistema de Ciencia y Tecnología en el sector y ejecución de acciones específicas para la formación de doctores. Las principales dificultades son: desmotivación de los profesionales para obtener el grado por las dificultades materiales que representa, escaso apoyo de las instituciones a los aspirantes, trato no diferenciado a los doctores en ciencias, ausencia de integración entre el pre y posgrado para la formación de doctores en ciencias, inexistencia de una cultura científica en el sector, carencia de presupuesto, definición bien establecida de las funciones de los tutores y poco uso de la intersectorialidad en la investigación.

Conclusiones El Sistema Nacional de Salud tiene posibilidades reales para la formación de doctores en ciencias de la salud. Las dificultades que atentan contra el proceso transitan desde la escasa motivación de los profesionales hasta otras de orden organizativo y de capacitación de los actores participantes. Estos problemas se solucionarán, en gran medida, cuando la formación de doctores en ciencias se encuentre considerada en la política de salud del país debidamente articulada con el Subsistema de Ciencia y Tecnología.

Palabras clave: Doctor en Ciencias, salud, formación, Cuba.

\section{ABSTRACT}

Objective To identify the favourable factors and the difficulties in the formation of doctors of philosophy (Ph.Ds) in health sciences.

Methods An exploratory study was conducted through in-depth interviews to 22 representatives of Scientific Boards of the Network of Cooperative Centers of the National School of Public Health for the formation of Ph.Ds in health sciences in institutions located in La Habana and in the medical universities of Santiago de Cuba y Camaguey. A brainstorming was held with representatives of the member centers of the network.

Results The favourable elements are the presence of a significant mass of doctors of philosophy in health sciences associated to the public health, availability of professionals having potentialities to reach the scientific degree, consolidation of the science and technology system in the sector and the implementation of specific actions for the formation of Ph.Ds. The main difficulties were lack of motivation on the part of professionals to attain the scientific degree, as a result of material difficulties, poor support by their institutions, lack of differentiated treatment to the Ph Ds, absence of integration between the undergraduate and postgraduate levels for the formation of these doctors, non-existence of a real scientific culture in the sector, lack of budget and of a well-established definition of the functions of tutors and poor use of the inter-sectorality in the research.

Conclusions The National Health Care System has real possibilities for the formation of $\mathrm{Ph}$ Ds in health sciences. The difficulties affecting this process range from the poor motivation of professionals to organizational and training problems found in the participating actors. These problems will be solved to a large extent when the formation of Ph Ds nationwide is taken into account in the domestic health policy and duly articulated with the science and technology subsystem.

Key words: Doctor in sciences, health, formation, Cuba. 


\section{INTRODUCCIÓN}

La Escuela Nacional de Salud Pública (ENSAP) desde su fundación se ocupó de desarrollar actividades y programas de posgrado, especialidades, cursos, entrenamiento, entre otros, campos en el que ha alcanzado un significativo desarrollo y prestigio, tanto nacional, como internacional, que ha sabido mantener hasta la actualidad. Una muestra que hace patente el avance de la institución son sus logros en el campo de la formación doctoral en Ciencias de la Salud.

El Doctorado en Ciencias de la Salud fue presentado al Pleno de la Comisión de Grados Científicos del Instituto Superior de Ciencias Médicas de la Habana, y a la Comisión Nacional de Grados en el año 1996 por la Facultad de Salud Pública, y se aprobó definitivamente en 1998, ya que se reconoció la pertinencia y necesidad de la propuesta, dentro del sector de la salud, pues el campo de investigación y acción de las Ciencias Médicas difiere del específico de las Ciencias de la Salud Pública, aunque es innegable su fuerte vinculación.

Al constituirse la ENSAP en el año 2002 resultó pertinente y oportuno diseñar y ejecutar una estrategia para acelerar la obtención del grado científico de Doctor en Ciencias de la Salud, así como solicitar la categoría de Institución Auspiciadora de la Academia de Ciencias, designación alcanzada en el año 2004. Como parte de esta estrategia y, para cumplir las orientaciones de los niveles superiores correspondientes, se actualizó el Programa de la Especialidad (2003), se elaboró el Programa de Doctorado Curricular Colaborativo y el Programa de Doctorado Tutelar en Ciencias de la Salud (2004-2005). Ambos programas, resultaron ser los primeros elaborados en el sector de la salud.

Para la ejecución del proceso de formación doctoral, como en otras instituciones autorizadas, se constituyó en diciembre de 2003 la Comisión de Grados Científicos de la ENSAP, integrada por 18 doctores, representantes de las diversas disciplinas de la Salud Pública. Esta Comisión: ha desarrollado con éxito sus funciones referidas a la aprobación, control y trámite de los procesos y las defensas de tesis para la obtención de grados científicos en Ciencias de la Salud, ha perfeccionado los procedimientos para la aceptación de los temas de tesis y la ejecución de los mínimos, ha logrado desarrollar un importante trabajo de coordinación con las Universidades Médicas y los Institutos Nacionales para realizar los ejercicios de predefensa y recientemente constituyó la Red de Centros Colaboradores de la ENSAP para la Formación de Doctores en Ciencias de la Salud, como una vía para perfeccionar el proceso de formación doctoral.

También se renovó el Tribunal Permanente de Ciencias de la Salud, integrado por 29 profesionales de diferentes instituciones del país vinculados con las Ciencias de la Salud, cuya sede es la propia ENSAP y que en el periodo 2004-2010 ha realizado 55 ejercicios de defensa de tesis doctorales en Ciencias de la Salud.

La ENSAP como institución autorizada para la formación posgraduada tiene el compromiso social de formar Doctores en Ciencias de la Salud en la propia institución, el resto del país y el mundo, para lo que cuenta con el Programa Tutelar y el Programa Curricular Colaborativo. Su ejecución se pauta por la estrategia general de formación que tiene el propósito de que los programas se lleven a cabo dentro del marco científico y académico que se requiere para garantizar la máxima calidad del proceso, le concede gran prioridad a la excelencia del claustro, la asesoría a todas las estructuras involucradas y el monitoreo y evaluación de todos los procedimientos. Este proceso transita desde la incorporación de nuevos candidatos, hasta la adquisición del grado científico (Estrategia General de Formación de Doctores en Ciencias de la Salud. ENSAP, 2010). 
La metodología para la formación de Doctores en Ciencias de la Salud que establece la estrategia contempla que la investigación para la obtención del grado científico responda a la solución de un problema de investigación priorizado del lugar donde trabaja el profesional y el seguimiento de dicha formación se hace mediante talleres de tesis en diferentes momentos: definición del problema de investigación, elaboración del proyecto, valoración de informe sobre los resultados parciales y final, de forma tal que el departamento y el centro participen y se responsabilicen con todas las etapas de la investigación. Estos talleres se realizan con la participación de Doctores en Ciencias y expertos en el tema del propio centro o invitados (Estrategia General de Formación de Doctores en Ciencias de la Salud. ENSAP, 2010).

El desarrollo exitoso de la estrategia demanda armonía del trabajo con el Sistema Nacional de Salud (SNS) que es donde se forman los aspirantes, sin embargo, los doctores en ciencias que han desarrollado la formación académica para la obtención del grado científico de Doctores en Ciencias de la Salud han percibido la existencia de dificultades presentes en el sector que entorpecen el proceso, por lo que se hace necesario su clara identificación, lo que constituye el propósito de la presente investigación.

Por otra parte, el desarrollo en la formación de doctores en Ciencias de la Salud y el reconocimiento que tiene la ENSAP dentro de las instituciones de salud y del propio ministerio ha sido la razón de haber sido encargada de la creación del soporte sistémico para impulsar la formación doctoral en el sector de la salud, para lo que serán muy importantes los resultados de la presente investigación.

\section{MÉTODOS}

Se realizó un estudio exploratorio con la utilización de técnicas cualitativas de recogida de información.

Los participantes fueron los representantes de la Red de Centros Colaboradores la ENSAP para formación de Doctores en Ciencias de la Salud.

Se aplicó una entrevista en profundidad a miembros de los 22 Consejos Científicos de la Universidad Médica de La Habana, institutos y centros de investigación y a las Universidades Médicas de Santiago de Cuba y Camagüey.

Se realizó una lluvia de ideas con los representantes de la Red de Centros Colaboradores para la Formación de Doctores en Ciencias de la Salud:

- Universidad Médica de La Habana.

- Facultad Enrique Cabrera.

- Facultad de Ciencias Médicas Julio Trigo.

- Facultad de Ciencias Médicas Finlay Albarrán.

- Facultad de Ciencias Médicas Manuel Fajardo.

- Facultad de Ciencias Médicas Calixto García.

- Facultad de Ciencias Médicas 10 de Octubre.

- Facultad de Ciencias Médicas Julio Trigo. 
- Facultad de Ciencias Médicas Miguel Enríquez.

- Instituto de Oncología y Radiobiología.

- Instituto de Nefrología.

- Centro de Desarrollo de la Farmacoepidemiología.

- Instituto de Medicina Tropical.

- Instituto de Nutrición e Higiene de los Alimentos.

- Instituto Nacional de Salud de los Trabajadores.

- Instituto de Hematología e Inmunología.

- Instituto de Endocrinología.

- Instituto de Gastroenterología.

- Instituto de Neurología y Neurocirugía.

La información obtenida se sometió a análisis de contenido, lo que permitió arribar a las categorías donde estuvieron enmarcados los factores favorables y dificultades que provocan la escasa formación de doctores en Ciencias de la Salud y permitió profundizar en las razones que las producen.

\section{RESULTADOS}

La formación de Doctores en Ciencias de la Salud transita por las mismas experiencias que las de otras especialidades. El sector ha tenido una consolidación en la actividad científica mediante programas y proyectos en el Subsistema de Ciencia y Tecnología, entre ellos, la Resolución No. 110 del Ministerio de Salud Pública (MINSAP) que contiene nuevas disposiciones en relación con la investigación científica, ${ }^{1}$ la Resolución No. 85 de 29 de mayo de 2003 de la Ministra de Ciencia, Tecnología y Medio Ambiente (CITMA) que establece el ordenamiento de los procesos de elaboración, organización, ejecución, financiamiento y control de los programas y proyectos de investigación científica e innovación tecnológica que forman parte del Sistema de Ciencia e Innovación Tecnológica, y el Reglamento del Sistema de Programas y Proyectos de Ciencia e Innovación Tecnológica, para normar la actividad de ciencia y tecnología de la salud, del MINSAP, y que se fundamenta en las dos Resoluciones antes mencionadas; es de Marzo de 2006 y está en fase de aprobación. Todas estas regulaciones y reglamentos armonizan con el eje medular para la obtención del grado científico que es la investigación. Se cuenta con Doctores en Ciencias de vasta formación científica y comprobado compromiso con la salud del país que trabajan en los programas doctorales creados, pero la cantidad es insuficiente y la edad es elevada.

Dentro de las oportunidades del Sistema Nacional de Salud para la formación de Doctores en Ciencias de la Salud y de otras especialidades se encuentra el hecho de contar con una masa de jóvenes profesionales con condiciones para ello, que se han preparado en su mayoría en el sistema de maestrías y especialidades del sector, lo que les permite abordar temas de investigación, dentro de los que se encuentran aquellos que demandan pocos recursos como son los de salud pública.

En algunas Universidades Médicas del país ya se han elaborado estrategias de formación de doctores de acuerdo a las necesidades de la salud y con énfasis en los jóvenes. Hay instituciones que han creado estructuras administrativas que se 
ocupan de la formación doctoral lo que demuestra la importancia que se le confiere a esta actividad en el momento actual.

En el caso específico de la ENSAP, se trabaja arduamente en la integración con las Universidades Médicas del país. Se organiza cada vez más el trabajo con la de La Habana, en cuyas facultades y servicios trabajan la mayoría de los aspirantes a Doctores en Ciencias de la Salud. Se ha logrado además un importante acercamiento a las Universidades Médicas de las provincias orientales, en particular Holguín y Guantánamo, también se han dado pasos de avances importantes en Cienfuegos, Villa Clara, Pinar del Río y Sancti Spíritus.

Los resultados no son aún suficientes, la edad promedio de los 38 graduados cubanos como Doctores en Ciencias de la Salud en la ENSAP en el periodo 2004-2010 es de alrededor de 50 años.

Otro elemento importante identificado es que los profesionales de la salud no se sienten motivados con la obtención del grado científico. Dentro de los factores subjetivos que pueden conducir a ello es que el tratamiento a los que ya son doctores en ciencias no se manifiesta en la estimulación y el trato en consonancia con lo que deben recibir. Son resaltadas dificultades objetivas que atraviesan los aspirantes para contar con los recursos necesarios, como por ejemplo: material de oficina, transportación, reactivos y otros insumos necesarios para la realización de las tesis. También se destacan los problemas con el acceso a Internet y la poca comprensión de las administraciones en el otorgamiento del fondo de tiempo necesario para la realización de la investigación, todo lo cual influye en la desmotivación que presentan los profesionales de la salud para la realización del doctorado.

Por otra parte, un aspecto señalado es que existe desconocimiento por parte de los profesionales interesados por el grado científico y los propios aspirantes sobre el compromiso social y la utilidad para la salud que traduce el contar con un título de Doctor en Ciencias. También es expresado con fuerza que el área estratégica del sector y las instituciones de salud no poseen la suficiente cultura científica que permita comprender la importancia de la investigación y dentro de la misma, la formación de doctores en ciencias, como instrumento fundamental para la solución de los problemas de la salud y la toma de decisiones. Se alude que esto pudiera ser la razón de la no existencia de un presupuesto diferenciado para la formación de doctores, dado que los gastos que se originan en este sentido pesan sobre otros rubros. De lo anterior pudiera plantearse que esto coadyuva a que muchos dirigentes administrativos e incluso los científicos no impulsan la formación de profesionales para la obtención del grado científico del área bajo su mando.

En todas las Universidades Médicas, Facultades de Ciencias Médicas, institutos y centros de investigación funcionan los Consejos Científicos organizadamente, no obstante en el trabajo desarrollado por la Comisión de Grados Científicos de la ENSAP ha identificado que aún prevalecen serios problemas relacionados con las actividades vinculadas con la formación de Doctores en Ciencias. Dentro de las principales dificultades detectadas se hallan el inadecuado monitoreo de los profesionales en proceso de doctorado que debe extenderse, desde que manifiestan interés por alcanzar el grado científico, hasta que lo obtiene. No está establecido el sistema de talleres de tesis en los departamentos donde labora el aspirante. También hay insuficiencias en la calidad de los proyectos que son avalados por los Consejo Científicos para el doctorado. 
Se ha identificado que cuando los Consejos Científicos son designados para la realización de las predefensas, hay insuficiencias en la conformación del colectivo con derecho al voto, lo que es, además, responsabilidad de la Comisión de Grados Científicos, en el llenado y tramitación de la documentación reglamentada y en el desarrollo del propio ejercicio.

Un elemento expuesto por los entrevistados y que ha conspirado en contra de la formación de los aspirantes a Doctores en Ciencias de la Salud, ha sido la inadecuada conducción de los tutores. La función del tutor no se encuentra claramente establecida y eso hace que se asuma por cada uno de acuerdo a su impronta personal. En muchas ocasiones, los tutores de tesis no tienen la experiencia necesaria en el tema que se investiga. El tutor es un doctor en ciencias que debe acompañar al aspirante en todo momento del proceso, que tiene a la investigación como eje central. Su actuación no puede ser solamente la revisión de un documento, sino la conducción del aprendizaje que demanda el aspirante para convertirse en un investigador. Debe velar porque el aspirante obtenga los conocimientos que necesita y tiene que brindar su experiencia en el tema que se investiga desde su génesis.

En la salud ha existido un divorcio entre la formación del profesional dirigida sobre todo a los servicios y a la creación de habilidades para la investigación. En general, los investigadores se han tenido que apropiar de dichas habilidades y conocimientos cuando se han enfrentado al trabajo en instituciones científicas; excepcionalmente, han existido profesionales, que trabajando directamente en el servicio han asumido la investigación y han logrado hacerse doctores en ciencias por propio interés personal. La formación de un investigador debe comenzar desde el pregrado donde se deben descubrir e iniciar la fragua de las posibilidades del individuo para esta actividad.

Desde hace un tiempo se ha impulsado la realización de maestrías en el campo de la salud, lo cual es un paso de avance en el pensamiento científico. En la ENSAP han sido 20 los graduados de las maestrías de la institución que han alcanzado el título de Doctores en Ciencias de la Salud. Pero, aún, los esfuerzos en este sentido no son suficientes en todo el país, por lo que es necesario implementar la continuidad entre los niveles de pregrado, la especialidad, maestría y el doctorado o entre niveles intermedios. El Doctorado en Ciencias de la Salud, en particular, demanda de la experiencia laboral del profesional relacionado sobre todo con la gestión del sistema y con el interés social de la salud, por lo que la continuidad se logra más en las etapas maestría-doctorado o en especialidad-maestría-doctorado, en este último caso cuando los profesionales son, en particular, especialistas de Bioestadística, Higiene y Epidemiología y Administración de Salud.

Existen centros de investigación de otras ramas de la ciencia con posibilidades reales de cooperación mutua para la investigación y la formación de doctores en ciencias de la salud que pueden complementar el conocimiento y el campo de acción de los temas tratados, no obstante se ha constatado que esta posibilidad real se encuentra poco aprovechada.

Los entrevistados señalan como un aspecto de gran importancia a tener en cuenta el intercambio académico en el área de doctorado con instituciones especializadas de otros países, lo que en la actualidad es escaso, sin embargo es muy importante en la toma de experiencias en el propio proceso de formación y las temáticas particulares que atiende el doctorado. 
Otra dificultad encontrada que atenta con la planificación y utilización de un recurso humano cuya preparación es costosa, es que no existe experiencia previa sobre la medición del impacto sobre el sistema que han tenido los graduados como Doctores en Ciencias de la Salud.

\section{DISCUSIÓN}

La formación de Doctores en Ciencias en el sector de la salud se tornó en una preocupación desde hace poco tiempo. En este sentido, se elaboró la estrategia general y el plan de acción. La ENSAP, como institución autorizada, ha realizado acciones específicas, una de las cuales es el trabajo realizado en Holguín que sirvió de experiencia para extenderlo al resto de las provincias. Se comprobó la posibilidad real de desarrollar el proceso de formación mediante la asistencia a las necesidades de los profesionales a través de cursos, asesorías y talleres, siempre que se contara con el respaldo de las autoridades sanitarias y docentes del territorio. $^{2}$

Es importante destacar que la estrategia general de doctorado que se desarrolla en la ENSAP muestra que son evidentes las ganancias en la calidad de los proyectos cuando se discuten en los talleres de tesis que se realizan en los departamentos donde trabajan los aspirantes. Esta práctica debe generalizarse a todas las instituciones de salud del país.

Las razones que conducen a las dificultades encontradas en el proceso de formación académica de Doctores en Ciencias de la Salud, que se extiende también a otras especialidades, pueden deberse a que, históricamente se incentivó la formación de especialistas en el sector para garantizar la calidad de la atención médica, y se postergó, en gran medida, la utilización de la investigación, y por tanto, la formación de doctores en ciencias como elemento trascendente en la solución de los problemas de la salud y para la toma de decisiones.

La formación de profesionales de la salud en Cuba tiene establecidas tres aristas: la asistencia, la docencia y la investigación, ,3 para lo que se encuentran en los programas docentes los contenidos que abordan cada una, sin embargo, la adquisición de conocimientos y habilidades para la investigación queda preterida, no está presente de forma transversal en la formación y tampoco se logra la conexión adecuada con el posgrado.

Las alianzas de trabajo en la investigación con ramas e instituciones relacionadas con la salud pública pueden favorecer la calidad de las mismas, sin embargo, la intersectorialidad en la investigación es poco explotada aún en la salud. ${ }^{5}$

La escasa preparación en investigación de los profesionales de la salud alcanza a los que se desempeñan como miembros de los Consejos Científicos. Estos órganos auxiliares de la dirección juegan un importante papel en el proceso de formación doctoral al tener la responsabilidad de avalar los proyectos de investigación y ejecutar ejercicios de gran connotación, como son las predefensas, que requieren de la selección de personas preparadas científicamente y vinculadas con el tema de tesis. Por otra parte, son los encargados de acompañar a los aspirantes a lo largo de su recorrido formativo, en la realización de los talleres de tesis y en la comprobación de los cronogramas y planes de trabajo de los aspirantes y en muchas ocasiones esta responsabilidad no es conocida y por lo tanto no es ejecutada. Por todo ello se impone realizar un proceso de capacitación a los Consejos Científicos en el área de la formación de Doctores en Ciencias de la Salud. 
Es importante puntualizar la función de los tutores, lo que debe ser objeto de análisis en las instancias de coordinación y conducción a nivel nacional y en la Comisión de Grados Científicos de la ENSAP.

El incremento del intercambio internacional en el área de la formación de Doctores en Ciencias de la Salud permitirá, por una parte, el enriquecimiento de los programas cubanos con las experiencias de otros países, además servirá de fuente de divulgación para la captación de extranjeros que quieran cursarlos y una forma muy efectiva de promover los logros de la salud pública cubana y de los resultados científicos alcanzados.

La medición del impacto de los graduados de formas académicas es un quehacer poco desarrollado en la salud, es por ello necesaria la creación de métodos para este fin. ${ }^{6}$ En el caso de los Doctores en Ciencias de la Salud, permitirá demostrar su utilidad y aporte al sistema, lo que no se conoce actualmente y será un trazador para la identificación de futuras necesidades de formación.

La formación de Doctores en Ciencias no puede ser el interés de una institución, sino debe formar parte de la política de salud del país, de forma integrada con el Sistema de Ciencia y Tecnología, que responda a las reales necesidades de la aplicación del método científico para la solución de los problemas existentes y por supuesto, que sirva de medio incuestionable en la formación científica de los aspirantes. Solo así se podrá realizar una planificación adecuada de actividades y recursos dirigidos a alcanzar un fin social compartido. La salud pública cubana no demanda de títulos, sino de profesionales altamente preparados para enfrentar las situaciones de alta complejidad que se presenten en el sector.

En general, el Sistema Nacional de Salud cuenta con posibilidades reales para la formación de Doctores en Ciencias de la Salud por haber consolidado el Sistema de Ciencia y Tecnología, contar con doctores en ciencias con alto nivel científico y compromiso con el sector e interés de las Universidades Médicas de realizar estrategias para la obtención del grado científico donde se involucren a los profesionales, sobre todo jóvenes en el abordaje de investigaciones dirigidas a la solución de los problemas de la salud del lugar donde trabajan.

Los aspectos favorables identificados para la formación doctoral pueden constituir elementos de apoyo para la formación de doctores en las diversas instituciones de salud del país, no obstante, se contraponen las dificultades identificadas, que atentan contra el proceso de formación de Doctores en Ciencias de la Salud y que responden a factores subjetivos, pero que son susceptibles de resolver si se trazan alianzas de trabajo efectivas entre las instituciones que participan en el proceso de formación doctoral desde diferentes perspectivas, todo ello enmarcado en los mecanismos institucionales y económicos vigentes.

La formación de Doctores en Ciencias de la Salud debe estar contenida en la política de salud del país debidamente articulada con el Subsistema de Ciencia y Tecnología, lo que permitirá la adopción de la cultura y la planificación adecuada de los recursos que demanda la formación de doctores en ciencias en el sector.

\section{REFERENCIAS BIBLIOGRÁFICAS}

1. Ministerio de Salud Pública (MINSAP). Resolución No. 110 de 24 de agosto de 2004. La Habana: Ministro de Salud Pública; 2004. 
2. Ochoa Alonso A. A, Rodríguez Cabrera A. Gestión organizacional para la formación doctoral local en Ciencias de la Salud en Holguín. Rev Cubana Salud Pública [Internet]. 2010 [citado 28 Jul 2011];36(3):280-4. Disponible en: http://scielo.sld.cu/scielo.php?script=sci arttext\&pid=S0864 $34662010000300018 \& \operatorname{lng}=\mathrm{es}$

3. Castañeda Abascal I, Rodríguez Cabrera A, Hernández Meléndez E, Rojo Pérez N. Universalización de la formación académica e investigativa para la obtención del doctorado en el sector salud. Rev Cubana Salud Pública [Internet]. 2008 [citado 28 Jul 2011];34(4). Disponible en:

http://scielo.sld.cu/scielo.php?script=sci_arttext\&pid=S0864 34662008000400015\&lng=es

4. Vidal Ledo M, Morales Suárez I. Universalización de la enseñanza. Educ Med Super [Internet]. 2005 [citado 28 Jul 2011];19(3). Disponible en: http://scielo.sld.cu/scielo.php?script=sci arttext\&pid=S0864$21412005000300010 \& \operatorname{lng}=\mathrm{es}$

5. Castell-Florit Serrate, Gispert Abreu E de los A. La intersectorialidad y el desarrollo de la Salud Pública en Cuba. Rev Cubana Salud Pública [Internet]. 2009 [citado 28 Jul 2011];35(1). Disponible en:

http://scielo.sld.cu/scielo.php?script=sci arttext\&pid=S0864-

34662009000100004\&lng=es

6. Couturejuzón González L, Columbié Pileta M. Impacto en la esfera intelectual del egresado de la especialidad de Bioestadística. Educ Med Super [Internet]. 2008 [citado 28 Jul 2011];22(4). Disponible en:

http://scielo.sld.cu/scielo.php?script=sci arttext\&pid=S0864 -

21412008000400004\&lng=es

Recibido: 31 de enero de 2011.

Aprobado: 17 de marzo de 2011.

Ileana Elena Castañeda Abascal. Escuela Nacional de Salud Pública. Calle 100 No. 10132 e/ Perla y E. Altahabana, Boyeros. La Habana, Cuba. Teléf.: 6438782 y 83, Ext. 137. Correo electrónico: ileca@infomed.sld.cu 